\title{
APPLICATION OF SWARM INTELLIGENCE ALGORITHM IN MECHANICAL FAULT DIAGNOSIS
}

\author{
Ding $M I^{1}, Y u L^{2} N^{2}$ \\ 1,2 Zhengzhou Shuqing Medical College, Hena, 450064, China \\ Email: dingmidm8812@163.com
}

\begin{abstract}
To solve the precision solution and velocity solution problems of complex functions with continuous variables in high dimensional continuous space, two kinds of newly improved swarm intelligence algorithms were presented, namely sinusoidal chaotic mapping fusing infinite collapse and SDLA improved algorithm of shrinkage factor in PSO algorithm - ISFLA. Through the analysis of $\mathrm{DE}$ algorithm, the individual update strategy in the algorithm was applied to the worst frog individual renewal process in the SFLA algorithm, to form a new strategy for multiple modes of evolution mutually competing existing at the same time. Moreover, a new method called MSFLA-CO was constructed. After the inertia weight of the worst frog update was set, a new improved algorithm based on inertial weight - MSFLA-Q was formed. The results showed that the effectiveness of the algorithm is verified by the simulation analysis of some test data sets and the examples of mechanical fault diagnosis. In a word, it is necessary to further perfect the fault diagnosis theory and method based on shuffled frog leaping algorithm and to integrate new fault diagnosis method.
\end{abstract}

Keywords: Fault Diagnosis; Swarm Intelligence Algorithm; Shuffled Frog Leaping Algorithm.

\section{Introduction}

Mechanical fault diagnosis is the science for the identification of running state of machines or units. Its core is the effective acquisition, transmission, processing, regeneration and utilization of diagnostic information, so as to possess the ability for accurate state recognition and diagnosis decision for diagnosis objects in a given environment, which is significant for protecting the safe operation of equipment (Lim et al., 2014). At present, with the mechanical equipment working condition complexity and large scale structure as well as function integration and automation development, how to extract useful information from these devices, judge the running state of the equipment and make an accurate fault diagnosis proposes a new test for the existing fault diagnosis method.

Artificial intelligence theories and methods are applied to the process of mechanical fault diagnosis, so that computers can do functions of reasoning, planning, decision making, learning and so on. Developing intelligent fault diagnosis is the main research direction at present (Sun et al., 2014; Pan et al., 2016). Heuristic optimization algorithm based on biological populations, such as evolutionary algorithms, genetic algorithms (GA), particle swarm optimization (PSO), immune algorithm and so on, usually called swarm intelligence algorithm. Its idea basis comes from swarm behaviour taken when natural creatures are foraging or conducting a certain activity. The swarm intelligence algorithm implementation is simple and efficient, and can be used to solve a large number of complex scientific and engineering problems (Sanchez et al., 2014; Duan et al., 2016; Zamani et al., 2017; Wang et al., 2017). The shuffled frog leaping algorithm (SFLA) is a kind of bionic optimization algorithm of swarm intelligence model, widely used in power system resources allocation optimization, intelligent transportation system optimization, industrial production scheduling and model parameter identification and so on. Whereas, it is seldom used in the field of mechanical fault diagnosis (Ladgham et al., 2015; Wang et al., 2017).

Based on this, according to the characteristics and actual situation of mechanical system fault diagnosis, the improved algorithm of SFLA was put forward, as a kind of new pattern recognition technology applied in the field of mechanical fault diagnosis.

\section{Methodology}

\subsection{Mechanical fault diagnosis}

The research content of equipment fault diagnosis involves fault mechanism, sensor and measurement, digital signal processing, modern control theory, reliability theory, mathematical statistics, fuzzy theory, pattern recognition, artificial intelligence, 
computer hardware and software and communication technology (Gomez et al., 2015).

From the point of view of engineering practice, the basic process is shown in Figure 1.

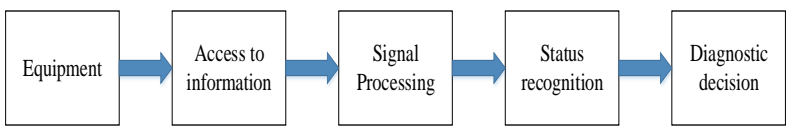

Figure 1. Mechanical fault diagnosis process diagram

We can see from Figure 1, the mechanical fault diagnosis mainly includes three basic processes: information acquisition, signal processing and state recognition. The essence of the first stage is to determine the sensors. According to the fault form, as well as monitoring parameters, monitoring site, monitoring methods and monitoring instrument sensitivity, it also determines type, number and position of the sensors, and correct and effective signal acquisition is a prerequisite for correct diagnosis. The essence of the second stage is to select and extract the feature information, which is the most important and most critical for the realization of fault diagnosis, but also one of the most difficult problems.

Most of the researches focus on the essence of this stage. The essence of the last stage is pattern recognition of equipment running state, which is another focus and hotspot of current studies.
Especially the development of grey theory, fuzzy theory, biomimetic intelligence and neural network in recent years makes it go step by step to the intelligent diagnosis (Lei et al., 2016).

\section{Shuffled frog leaping algorithm}

SFLA, as the new bionics intelligent group optimization algorithm, adopted hybrid multi population evolution (Shuffled Complex EvolutionUniversity of Arizona, SCE-UA or SCE) algorithm as the breadth search implementation framework. Meanwhile, it is combined with memetic algorithm (MA) for local search in the PSO algorithm. In essence, SFLA is swarm intelligent optimization algorithm (Mahmoudi et al., 2016), which combines deterministic competitive evolutionary strategy and finite random search.

The deterministic strategy makes the algorithm effectively guide the algorithm going forward on the right direction according to some information, and the random search algorithm can improve the robustness and adaptability. The formula (1) describes the SFLA allocation process, and $\mathrm{Y}^{\mathrm{k}}$ is a collection of $k$-th frog population, $\left.j=1, \ldots, n_{p}\right], k=1, \ldots$, $\mathrm{m}_{\mathrm{p}}$ :

$$
Y^{k}=\left[U(j)^{k}, f(j)^{k} \mid U(j)^{k}=U\left(k+m_{p}(j-1)\right), \mathrm{f}(j)^{k}=f\left(k+m_{p}(j-1)\right)\right.
$$

During the iteration, in each population, $\mathrm{q}$ frogs were selected to construct a subgroup. The worst frog $U_{w}$, the best frog $U_{b}$ and the global best frog $U_{g}$ of the whole group were identified. Then $U_{w}$ was updated, and the update strategy was:

$$
\begin{aligned}
& S=\left\{\begin{array}{l}
\min \left\{\operatorname{int}\left(r\left(U_{b}-U_{w}\right)\right), S_{\max }\right\} U_{b}-U_{w} \geq 0 \\
\max \left\{\operatorname{int}\left(r\left(U_{b}-U_{w}\right)\right),-S_{\max }\right\} U_{b}-U_{w}<0
\end{array}\right. \\
& U_{w}^{\prime}=U_{w}+S
\end{aligned}
$$

In the formula, $\mathrm{S}$ is the worst frog adjustment vector; min and max are the minimum and maximum function, respectively; int suggests the integral function; $r$ is a random number between $[0,1]$; $S_{\text {max }}$ represents the maximum step size allows the frog to change.

Table 1. Comparison of Lyapunov Exponents of Different Chaotic Maps

\begin{tabular}{|c|c|c|c|c|}
\hline Mapping method & This article & $\begin{array}{c}\text { Related } \\
\text { documents }\end{array}$ & Logistic map & Chebyshev map \\
\hline Lyapunov index $\lambda$ & 3.6777 & 1.6696 & 0.6940 & 1.3868 \\
\hline
\end{tabular}

In order to make full use of the information of $\mathrm{U}_{\mathrm{b}}$ and $U_{g}$ and accelerate the convergence time of the algorithm, we propose a particle swarm update strategy with shrinkage factor, which improves the update strategy of the worst frog in SFLA, as shown

\section{Improvement of shuffled frog leaping algorithm}

When solving low dimensional problems, SFLA has some advantages in performance compared with similar intelligent optimization algorithms. However, with the increasing complexity and dimension of solving problems, the accuracy and convergence speed of SFLA will also decrease, as shown in Table 1. In order to improve the accuracy of the solution and increase the number of frog population or the number of global iterations, it is inevitable that the calculation cost of the algorithm will be greatly improved. To overcome this shortcoming, this paper proposes an improved algorithm based on chaos theory and convergence factor, abbreviated as ISFLA. 


$$
U_{w}^{\prime}=k \cdot\left(U_{w}+S\right)
$$

BP network is a kind of supervised learning method, and it is one of the most widely used neural network models.
Figure 2 is a typical topology of a three-level BP neural network model, including the input layer composed of i neurons, the hidden layer composed of $\mathrm{j}$ neurons, and the output layer composed of $\mathrm{k}$ neurons.

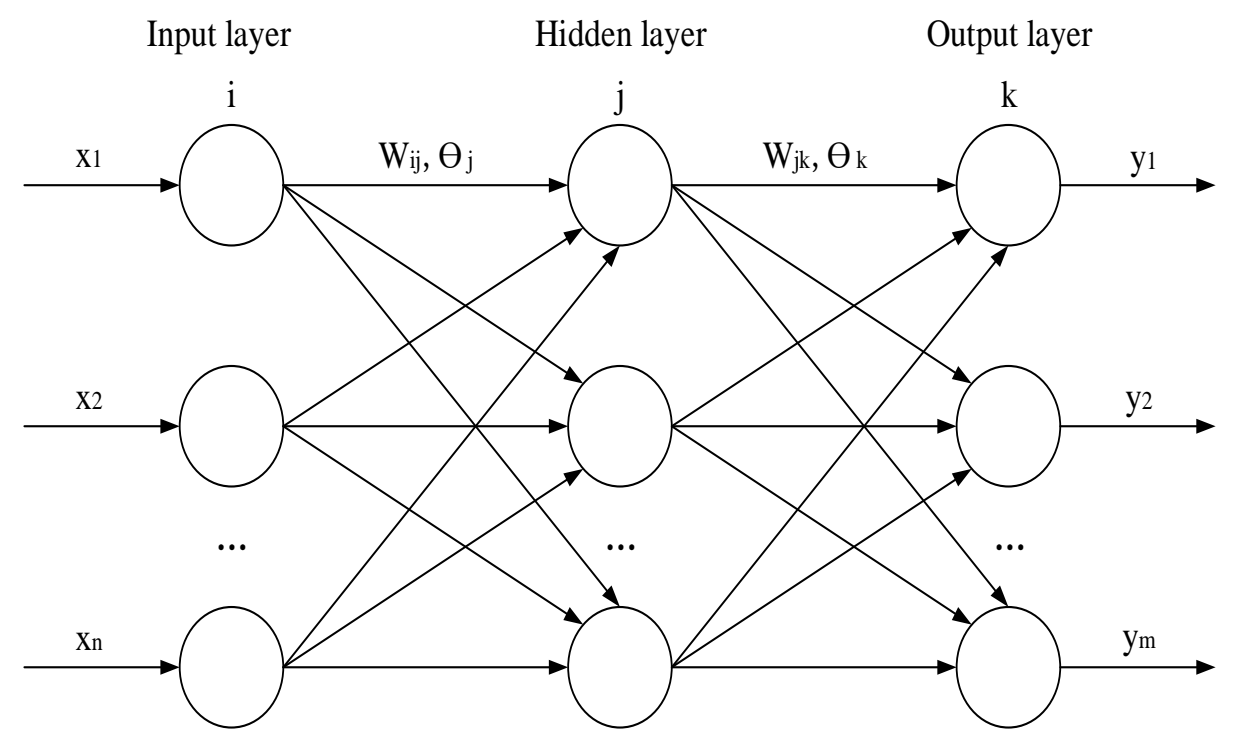

Figure 2. Three-layer BP network model

DE algorithm is a typical swarm intelligence algorithm characterized by memory individual optimal solution and information sharing in population.

That is, through cooperation and competition among individuals in the population, solution to the optimization problem is realized and its essence is a kind of greedy genetic algorithm with ensuring quality of thought (Rashidi et al., 2015).

The iterative process of the DE algorithm is shown in Figure 3.

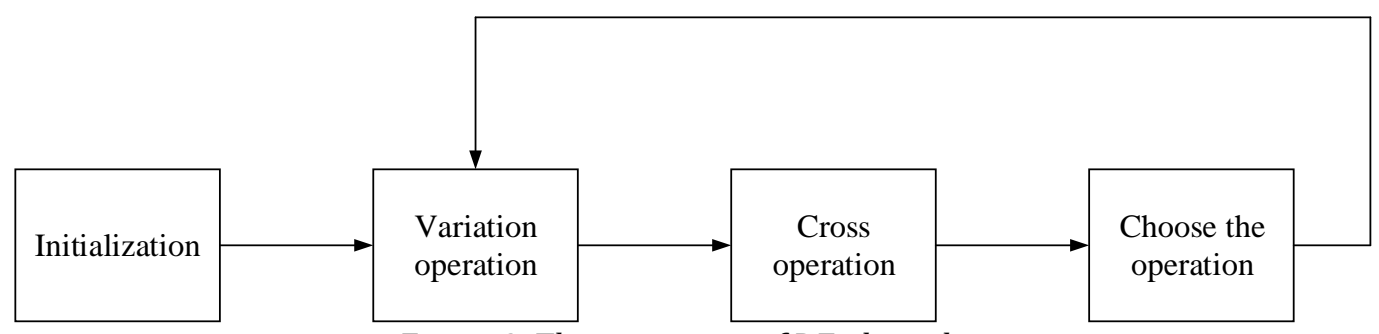

Figure 3. The main steps of DE algorithm

One of the most obvious characteristics of SFLA different from other swarm intelligence algorithms is: in the whole process of evolution, only the worst frog in the sub group is updated not all frogs. In addition, under ideal circumstances, the updated frog should increase and inherit more excellent frog characteristics, and minimize the impact of the worst frog at the same time. Based on this, the original leapfrog rules are introduced with a new parameter of inertia weight $\alpha$ and a modified SFLA based on inertia weight is formed, abbreviated as MSFLA. The old and new frog leaping rules are shown as follows:

$$
U_{w}^{\prime}=\alpha U_{w}+S
$$

Vector graphics of standard SFLA frog leaping rules is shown in Figure 4 (a) and vector graphics of new frog leaping rules based on inertia weight MSFLA is shown in Figure 4 (b). It can be clearly seen that, the new rules can broaden the worst frog jumping direction and also effectively increase (or shorten) jump step length. As a result, the search space is greatly improved; the worst frog has higher probability to get rid of the disadvantageous position; the performance better than the best frog before this update is obtained. Therefore, in theory, the modified algorithm can effectively avoid the premature phenomenon and improve the performance of the algorithm. 


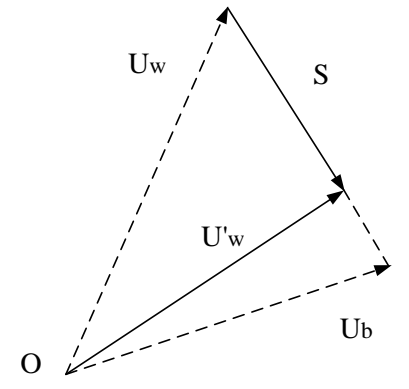

(a) The original frog jump rules

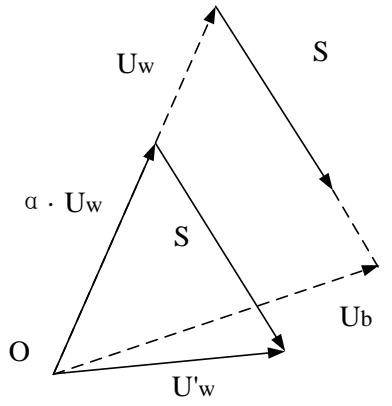

(b) Improved leapfrog rule

Figure 4. Frog jumping rules vector illustration

On the basis, the three kinds of inertia weight forms are proposed, to form different modified algorithms. They are modified shuffled frog leaping algorithm based on random inertia weight (MSFLA$\mathrm{R})$, modified shuffled frog leaping algorithm based on linear decreasing inertia weight (MSFLA-L) and modified shuffled frog leaping algorithm based on nonlinear decreasing inertia weight (MSFLA-Q), respectively.

Finally, all the improved algorithms proposed in this paper are simulated and analyzed to evaluate their performance. At the same time, it is applied in the field of mechanical equipment fault diagnosis.

\section{Results and Discussion}

3.1 Improvement and application of continuous shuffled frog leaping algorithm based on chaos theory and shrink3. age factor

In order to objectively evaluate the performance of the modified algorithm, we use the two typical test functions of Sphere function $\left(\mathrm{F}_{1}\right)$ and Ackley function $\left(\mathrm{F}_{5}\right)$ to test ISFLA and compare it with the result of standard SFLA. The Sphere function is a relatively simple single peak function, and the Ackley function is a complex multi-peak function.

Figure 5 and Figure 6 gives the evolution curve when Sphere and Ackley function solves the minimum value. Because the function value of the two is small, in order to facilitate viewing, the fitness value is conducted with logarithm value with the constant 10 as the bottom. It can be seen that, in the same number of global iterations, ISFLA was higher than that of SFLA. With the increase in the number of iterations, the convergence precision of the Sphere function will improve, until it reaches the limit of computer memory. In the solution of the Ackley function, when $\mathrm{N}=132$, the algorithm has completely and stably convergent on the approximate global optimum. Thus, improved shuffled frog leaping algorithm - ISFLA has good adaptability, stability and rapid global search capability.

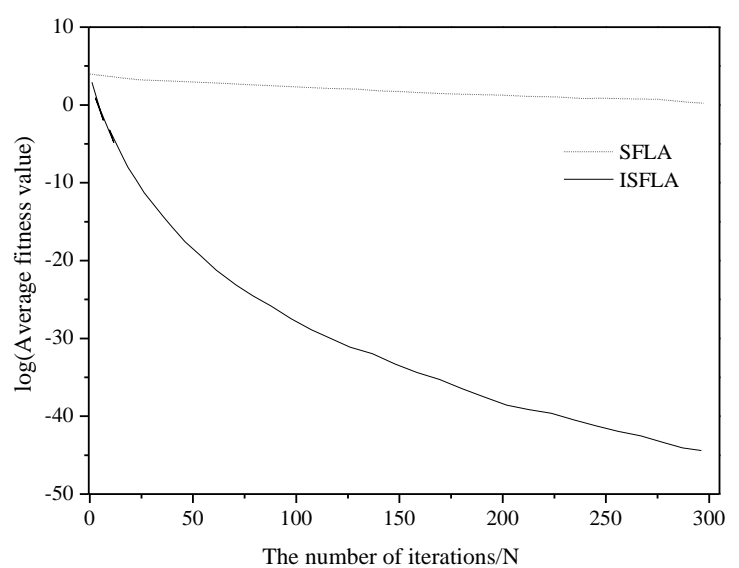

Figure 5. Sphere function evolution curve

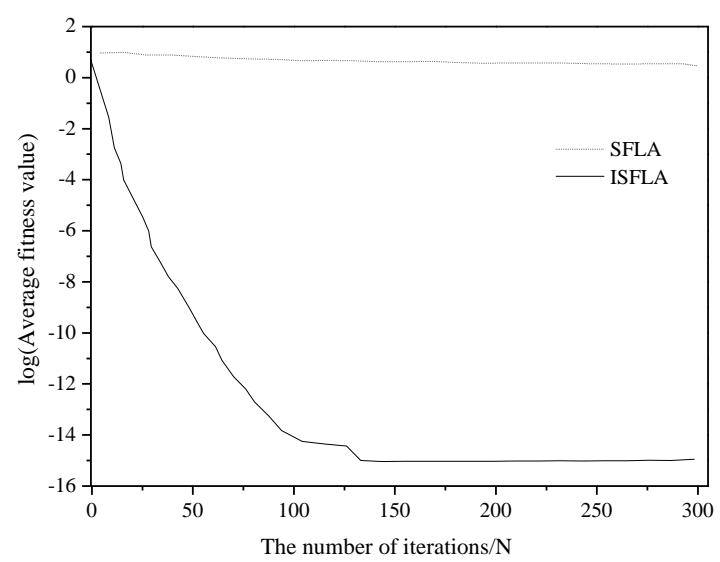

Figure 6. Ackley function evolution curve

We used the fault data in the rolling bearing fault simulation test bench driving end of Electrical Engineering Laboratory of Case Western Reserve University, selected the bearing signals under normal state and 5 bearing signals with early fault as fault mode set. $\mathrm{fm}_{1}$ represents the normal state; $\mathrm{fm}_{2}$ and $\mathrm{fm}_{3}$ are the inner faults with the damage diameter of $0.1776 \mathrm{~mm}$ and $0.3555 \mathrm{~mm}$, respectively; $\mathrm{fm}_{4}$ and $\mathrm{fm}_{5}$ are roller damages with the damage diameter of $0.5335 \mathrm{~mm}$ and $0.7113 \mathrm{~mm}$, respectively; fm6 suggests the outer ring injury with the damage diameter of 1.017 . 
In order to compare the performance of the method, BP network, SFLA-BP network and ISFLA-BP network are used to diagnose the early faults of six kinds of bearings.

The recognition rate results of three kinds of networks for 6 kinds of bearing of a total of 150 groups testing samples are shown in Figure 7 . We can see that for the rolling bearing of normal state $\left(\mathrm{fm}_{1}\right)$ and outer injury $\left(\mathrm{fm}_{6}\right)$, the recognition rate of three kinds of networks is all above $90 \%$; and for the slight early inner damage $\left(\mathrm{fm}_{2}\right)$ and the roller damage $\left(\mathrm{fm}_{4}\right)$, the recognition rate of BP network is the lowest, below $80 \%$. Especially the rolling bearing in a serious injury ( $\mathrm{fm} 5$ ), the recognition rate is only $43.62 \%$. This is because the BP network trapped into a local optimum and precocious in the search for the best network weights and threshold value, leading to decrease of learning ability. After using SFLA and ISFLA for the optimization of BP network parameters, compared with the initial parameter values randomly selected, it can improve the recognition rate of the network, of which ISFLA-BP has the best effect.

Therefore, the recognition rate of all kinds of bearing status has reached over $84 \%$, and the overall recognition rate has reached $89.35 \%$. Compared with BP network and SFLA-BP network, the overall mean recognition rate has increased by $11.45 \%$ and $4.68 \%$, respectively.

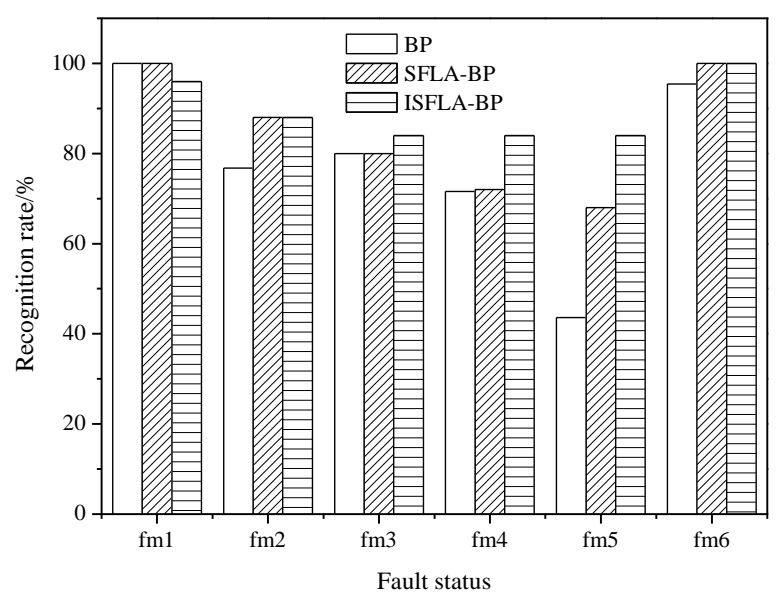

Figure 7. Comparison of three networks recognition rate

\subsection{Exploration of SFLA improvement based on competition strategy and its application in adaptive clustering analysis}

A modified SFLA based on the coexistence of update strategy and mutual competition is abbreviated as MSFLA- $\mathrm{C}_{0}$. The basic idea is, when the standard SFLA updates the worst frog, to keep updating strategy in the original algorithm, but also introduce the mutation and crossover strategy for individual update in DE algorithm, so as to form a plurality of update values of the worst frog in the sub population. Then, the frog values with different update strategies are compared to select frogs with the best fitness value as the worst frog update value. In order to evaluate the performance of the improved algorithm, we use the two typical test functions of Sphere function $\left(F_{1}\right)$ and Ackley function $\left(\mathrm{F}_{5}\right)$ to test MSFLA-CO and compare it with the results of other algorithms.

Figure 8 and Figure 9 is the curve of, average convergence curve varying with global iterations for two test functions in different algorithms. For the two test functions, both in convergence accuracy and convergence speed, the performance of SFLA and MSFLA-CO was better than DE algorithm. At the same time, MSFLA-Co is better than the standard SFLA among them. In Figure 11, A is the convergence point of convergence curve. After the convergence curve passes this point, it is completely convergent to the theoretical global best point of test function - 0 point.

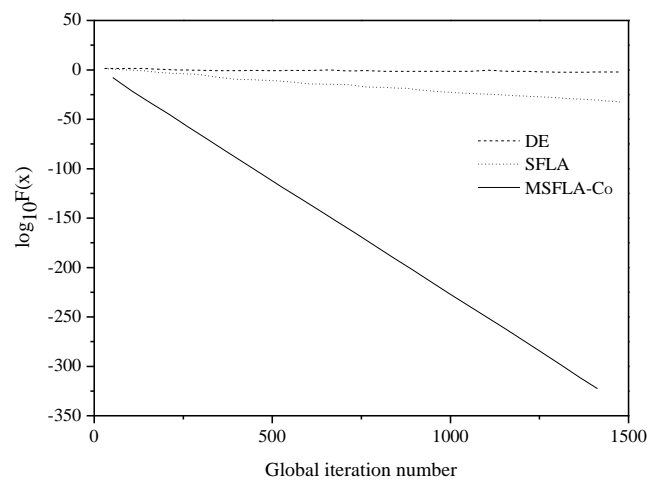

Figure 8. The average convergence curve for Sphere function $\left(F_{1}\right)$

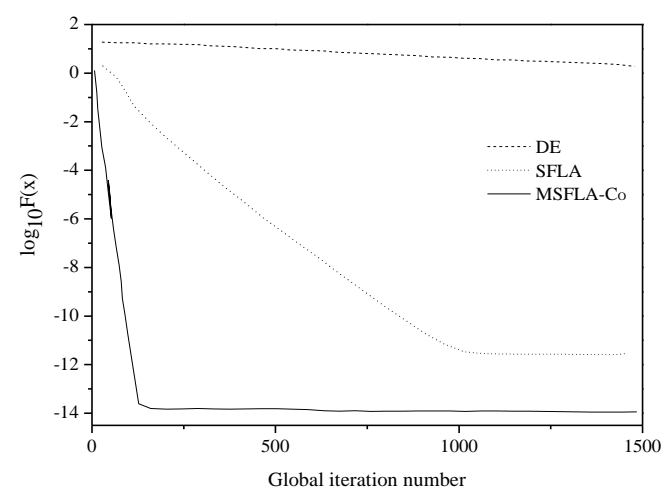

Figure 9. The average convergence curve for Ackley function $\left(F_{5}\right)$

Through an artificial data set and Iris data set of a UCI database, the simulation experiment verification of the proposed CA-MSFLA-CO is carried out. First of all, the best number of clusters is solved for the data set. The cluster analysis method based on SFLA and MSFLA-CO is applied, respectively.

Then, the clustering center of each data set is calculated and compared with the result calculated by K-Means method. The result is shown in Figure 10 . 


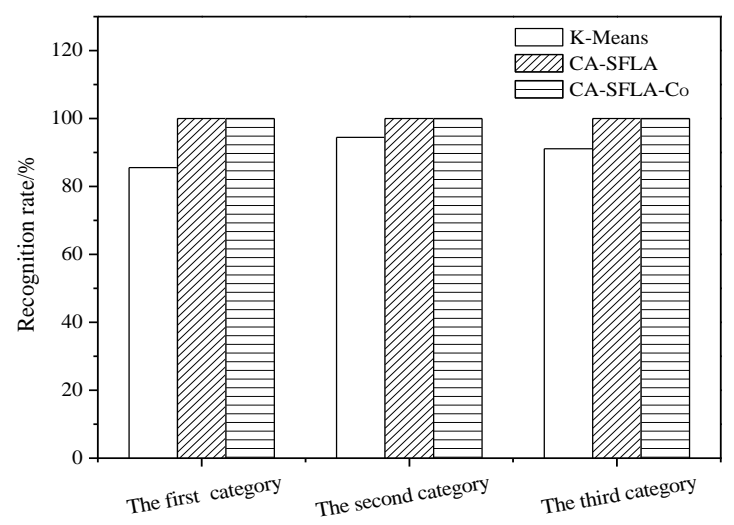

Figure 10. Comparison of the results of the three algorithms

From the result of recognition rate, for this artificial data, the two algorithms proposed in this paper can reach $100 \%$ by 10 independent operations, while the result of $\mathrm{K}$-Means operation is different. Although most of them can achieve 100\% accuracy, there is also a recognition error. In theory, the three clustering center vector values have great error, due to the existence of recognition error, in the actual calculation process. The proposed clustering center of modified clustering algorithm MSFLA-CO is different, but relatively stable, so as to prove the effectiveness of the proposed algorithm.

In this paper, the vibration data of a gearbox test rig in the laboratory are analyzed, and the state types of gearbox are divided into three parts, namely normal state $\left(\mathrm{fm}_{1}\right)$, uniform wear fault state $\left(\mathrm{fm}_{2}\right)$ and local wear (pitting corrosion) fault state ( $\left.\mathrm{fm}_{3}\right)$.

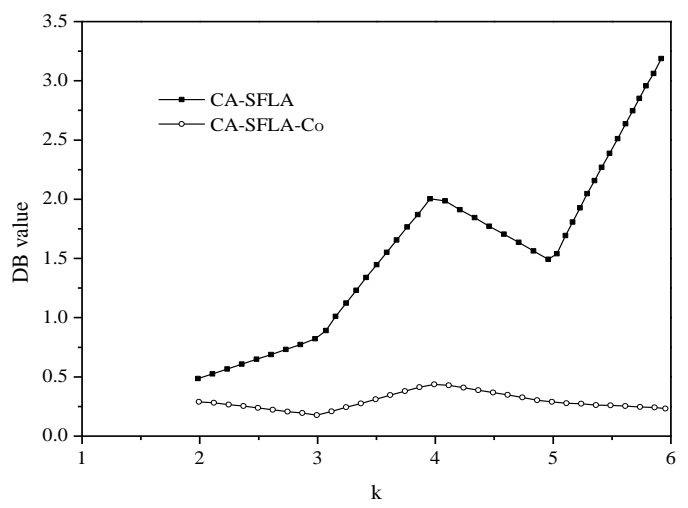

Figure 11. Gearbox data set DB-K diagram

It can be seen from Figure 11 that, the best clustering number found by CA-SFLA algorithm is $\mathrm{K}^{*}=2$, which is not consistent with the state type of actual gear box. The further observation results show that the algorithm separates the normal state and fault state, but the fault between them cannot be separated. In this paper, the method of clustering analysis based on MSFLA-Co can accurately find out the optimal clustering number of this data set $\mathrm{K}^{*}=3$, fully consistent with the actual situation.
At the same time, in order to illustrate the effectiveness of the algorithm, when $K^{*}=3$, the $K$ Means algorithm mentioned processes the gear box data. The calculation is compared with the two algorithms. In order to overcome the influence of accidental error, the three algorithms are run independently for 10 times and the average values are compared. Among them, the recognition rate of all samples is shown in Figure 12.

Therefore, in the data analysis on the clustering of such complex data, the recognition rate of CAMSFLA-CO is the highest.

Although the overall recognition rate of K-Means algorithm reached $87.5 \%$, the 10 times operational results show that the influence of different initial clustering centers on K-Means algorithm is great and the recognition results are not stable than the algorithm proposed in this paper.

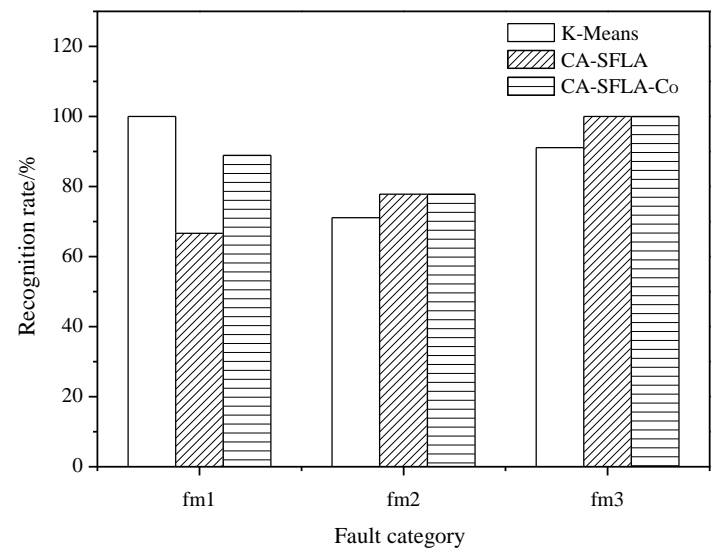

Figure 12. Comparison of the recognition rate of the three algorithms

\subsection{Improvement and application of shuffled frog leaping algorithm based on inertia weight}

In order to evaluate the performance of the improved algorithm, we use the two typical test functions of Sphere function $\left(F_{1}\right)$ and Ackley function $\left(\mathrm{F}_{5}\right)$ to test MSFLA and compare it with the result of standard SFLA. Figures 13 and Figure 14 are curves of the average convergence curve varying with the number of global iterations for two test functions under different algorithms.

For example, for the convenience of observation, the logarithmic scale with 10 as the bottom is conducted on the ordinate (average optimal solution).

Among them, A, B and C are 3 convergence points in the convergence curves of three improved algorithms. It indicates that the convergence curve is completely convergent to the theoretical global optimum - 0 point after passing the point. 


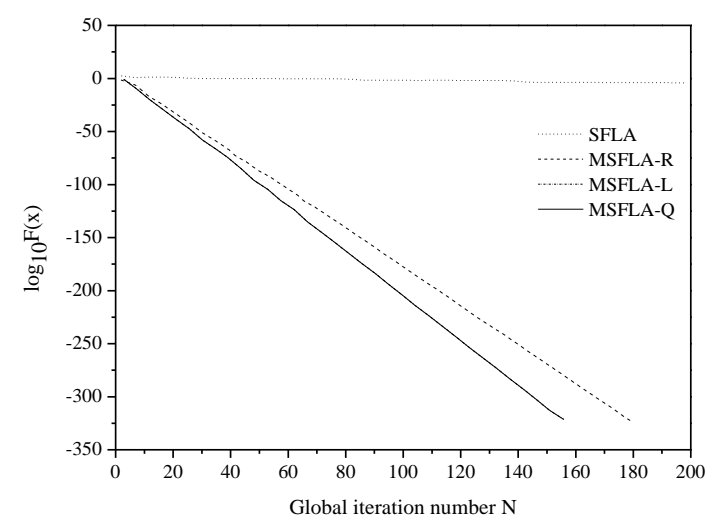

Figure 13. The average convergence curve for Sphere function $\left(F_{1}\right)$

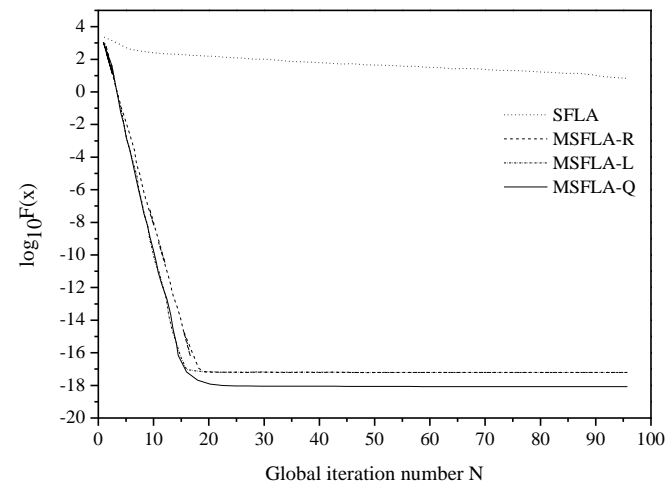

Figure 14. The average convergence curve for Ackley function $\left(F_{5}\right)$
It can be seen from the chart that, for the two test functions, 3 kinds of modified MSFLA algorithm are better than the original SFLA both in convergence accuracy and convergence speed, especially the three algorithms are all completely convergent to global optimal solution in theory, and the convergence speed is far greater than that of SFLA under the same conditions. In these 3 kinds of improved algorithms, the performance of MSFLA-Q is similar to MSFLA-L and better than MSFLA-R. In particular, in the early stages of solving $F_{5}$ function, MSFLA-L and MSFLA-Q basically coincide. But in the later period, MSFLA-L and MSFLA-R overlap together and its accuracy is slightly lower than that of the MSFLA-Q algorithm.

To sum up, the above three MSFLA algorithms based on different inertia weight strategies are better than the standard SFLA in terms of stability, adaptability and algorithm convergence.

The process of mechanical fault diagnosis based on MSFLA-VPMCD can be expressed in Figure 15. In order to verify the effectiveness of the fault diagnosis method based on MSFLA-VPMCD, this paper uses the fault data of rolling bearing fault simulation test bench driving end of Electrical Engineering Laboratory in Case Western Reserve University.

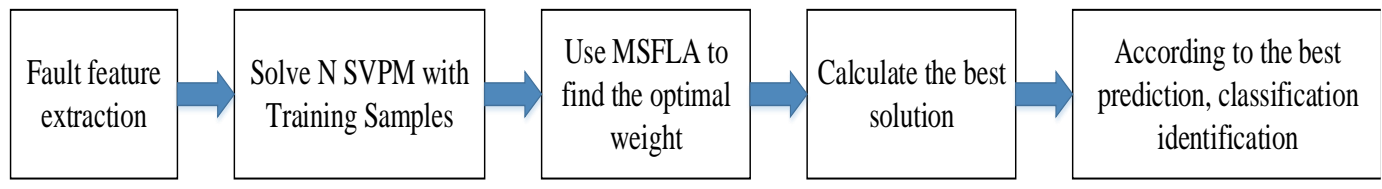

Figure 15. MSFLA-VPMCD fault classification flow chart

The state models of rolling bearing were normal $\left(\mathrm{fm}_{1}\right)$, inner ring damage $\left(\mathrm{fm}_{2}\right)$ outer ring damage $\left(\mathrm{fm}_{3}\right)$ and roller damage $\left(\mathrm{fm}_{4}\right)$ a total of 4 kinds.
The correct rate of classification recognition for VPMCD and MSFLA-VPMCD is shown in Table 2.

Table 2. Recognition rate (\%) comparison of each model in the VPMCD and MSFLA-VPMCD methods

\begin{tabular}{|c|c|c|c|c|c|}
\hline & \multicolumn{4}{|c|}{ VPMCD } & \multirow{2}{*}{ MSFLA-VPMCD } \\
\cline { 2 - 5 } & VPM(1,4) & VPM(2,4) & VPM $(3,4)$ & VPM $(4,4)$ & 100 \\
\hline $\mathrm{fm}_{1}$ & 100 & 100 & 100 & 100 & 92.5 \\
\hline $\mathrm{fm}_{2}$ & 64.3 & 72.4 & 64.4 & 68.5 & 88.6 \\
\hline $\mathrm{fm}_{3}$ & 56.1 & 60.5 & 64.2 & 60.3 & 96.9 \\
\hline $\mathrm{fm}_{4}$ & 84.2 & 92 & 91.9 & 91.8 & 94.5 \\
\hline Average & 76.2 & 81.2 & 80.1 & 80.1 & \\
\hline
\end{tabular}

Table 2 shows, for the rolling bearing in the normal state $\left(\mathrm{fm}_{1}\right)$, both the 4 variables prediction model VPM of standard algorithm and the best variables prediction model in the proposed MSFLAVPMCD, it can correctly identify; while for the state pattern of the inner ring damage $\left(\mathrm{fm}_{2}\right)$ and the outer ring damage $\left(\mathrm{fm}_{3}\right)$, no matter which VPM is, the recognition rate is worse, the highest is only $72.4 \%$ and the lowest is only $56.1 \%$. But for the best variables prediction model of MSFLA-VPMCD, the recognition rate is greatly improved, reaching $92.5 \%$ and $88.6 \%$, respectively. Although it did not reach $100 \%$, it may be caused by the internal relationship of the rolling bearing early fault features amount; for roller damage $\left(\mathrm{fm}_{4}\right)$ model, two algorithms have maintained higher recognition rate. 
Compared with VPMCD of single variable prediction model, the effectiveness, generalization ability and robustness of MSFLA-VPMCD are obviously improved.

\subsection{Performance comparison of three continuous variables modified shuffled frog leaping algorithms}

In order to compare the difference of three kinds of modified shuffled frog leaping algorithms for continuous variables, two different types of Benchmark function proposed in the previous paper are tested. For better observing the actual performance of various modified algorithms in high dimensional space, the test dimension is number of variables) $\mathrm{D}=100$.

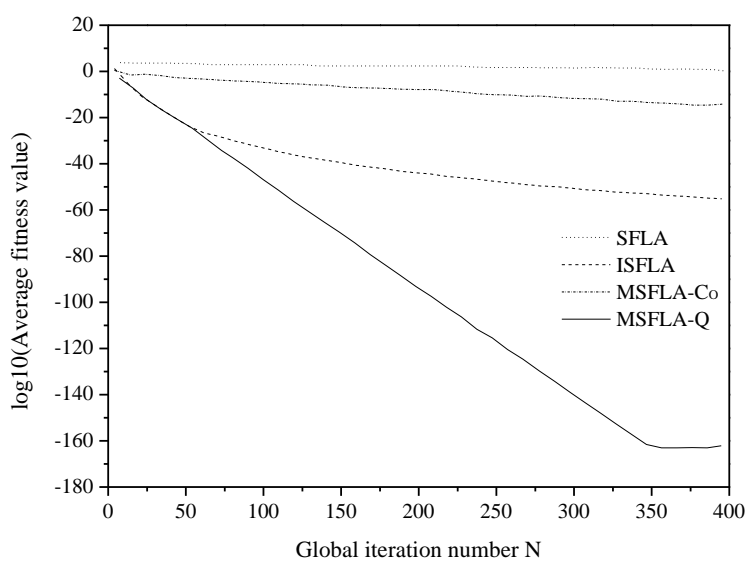

Figure 16. $F_{1}$ function of the average convergence curve

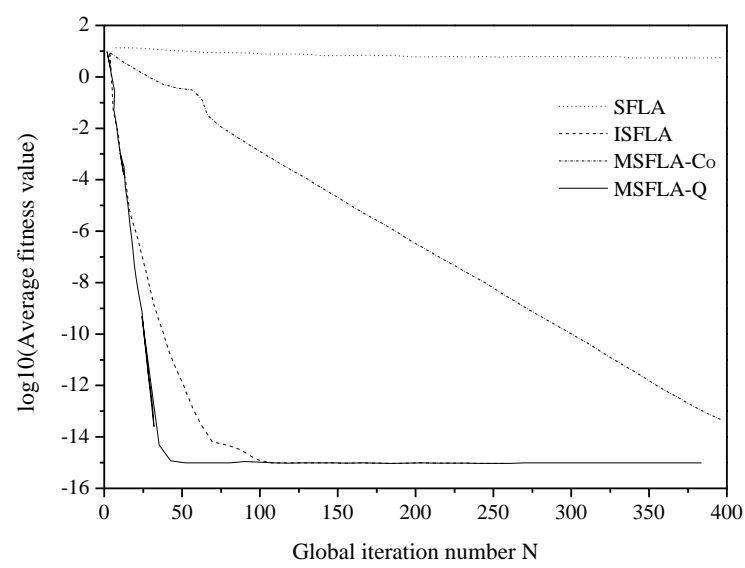

Figure 17. $F_{5}$ function of the average convergence curve

Figures 16 and Figure 17 illustrate the different speed of four algorithms in solving different test functions under the same number of global iterations. It can be seen that although the solution accuracy of ISFLA and MASFLA-Q is similar, the solution speed is quite different. The speed of the latter is better than that of the former, but the two are faster than the MSFLA-CO algorithm. The simulation results showed that the three improved methods had their own characteristics and could be selected flexibly in practical engineering applications.

\section{Conclusion}

In view of the deficiency of standard SFLA and some problems in the fault diagnosis of mechanical equipment, the new swarm intelligence algorithm -shuffled frog leaping algorithm and improved algorithm was applied in fault diagnosis of machinery. From the "intelligent optimization" processing point of view, we completed the parameter optimization of neural network model in diagnosis system and smart solution to the cost function and the number of clusters in unsupervised machine learning. Three modified algorithms for continuous variables were proposed, namely modified algorithm based on chaos theory and shrinkage factor, modified algorithm based on competitive strategy and modified algorithm based on inertia weight. Moreover, the convergence performance of three kinds of modified SFLA was compared. The experimental results of two simulation test functions showed that the three improved methods had different characteristics and could be combined in the practical application in mechanical fault diagnosis.

Due to the complex mathematical theory related to swarm intelligence algorithm, involving multidisciplinary crossover and fusion, both in theory and practice, researches on fault diagnosis based on SFLA are insufficient. The theory and method of fault diagnosis based on shuffled frog leaping algorithm needed to be further perfected and new fault diagnosis method was fused. In summary, research on engineering application of the fault diagnosis method based on SFLA should be carried out, and especially the engineering application of large-scale and complex mechanical system expands the new application scope of fault diagnosis.

\section{References}

[1] Lim, G. M., Bae, D. M., Kim, J. H. (2014). Fault diagnosis of rotating machine by thermography method on support vector machine. Journal of Mechanical Science and Technology, 28(8): 29472952.

[2] Sun, H., He, Z., Zi, Y., et al (2014). Multiwavelet transform and its applications in mechanical fault diagnosis-a review. Mechanical Systems and Signal Processing, 43(1): 1-24.

[3] Pan, J., Chen, J., Zi, Y., et al (2016). Monocomponent feature extraction for mechanical fault diagnosis using modified empirical wavelet transform via data-driven adaptive Fourier 
spectrum segment. Mechanical Systems and Signal Processing, 72: 160-183.

[4] Sanchez, V. M., Chavez-Ramirez, A. U., DuronTorres, S. M., et al (2014). Techno-economical optimization based on swarm intelligence algorithm for a stand-alone wind-photovoltaichydrogen power system at south-east region of Mexico. International journal of hydrogen energy, 39(29): 16646-16655.

[5] Duan, S. M., Mao, J. L., Li, J. L., et al (2016). Design implementation and application of swarm intelligence algorithm optimization function simulation platform. Software Engineering and Information Technology: Proceedings of the 2015 International Conference on Software Engineering and Information Technology (SEIT2015): 196-203.

[6] Zamani, M., Khairuzzaman, M., Musirin, I., et al (2017). Multi-area economic dispatch performance using swarm intelligence technique considering voltage stability. International Journal on Advanced Science, Engineering and Information Technology, 7(1): 1-7.

[7] Ladgham, A., Hamdaoui, F., Sakly, A., et al (2015). Fast MR brain image segmentation based on modified Shuffled Frog Leaping Algorithm. Signal, Image and Video Processing, 9(5): 1113-1120.
[8] Wang, L., Guo, C., Guo, S., et al (2017). Rescheduling strategy of cloud service based on shuffled frog leading algorithm and Nash equilibrium. The International Journal of Advanced Manufacturing Technology: 1-17.

[9] Gomez, A. J. F., Sobczyk, T. J., Weinreb, K. (2015). Influence on rotor broken bar fault diagnosis of mechanical torque pulsations by means of FFT. Diagnostics for Electrical Machines, Power Electronics and Drives (SDEMPED), International Symposium on. IEEE: 44-49.

[10] Lei, Y., Jia, F., Lin, J., et al (2016). An intelligent fault diagnosis method using unsupervised feature learning towards mechanical big data. IEEE Transactions on Industrial Electronics, 63(5): 3137-3147.

[11] Mahmoudi, N., Orouji, H., Fallah-Mehdipour, E. (2016). Integration of Shuffled Frog Leaping Algorithm and Support Vector Regression for Prediction of Water Quality Parameters. Water resources management, 30(7): 2195-2211.

[12] Rashidi, F., Abiri, E., Niknam, T., et al (2015). Parameter identification of power plant characteristics based on PMU data using differential evolution-based improved shuffled frog leaping algorithm. International Journal of Bio-Inspired Computation, 7(4): 222-239.

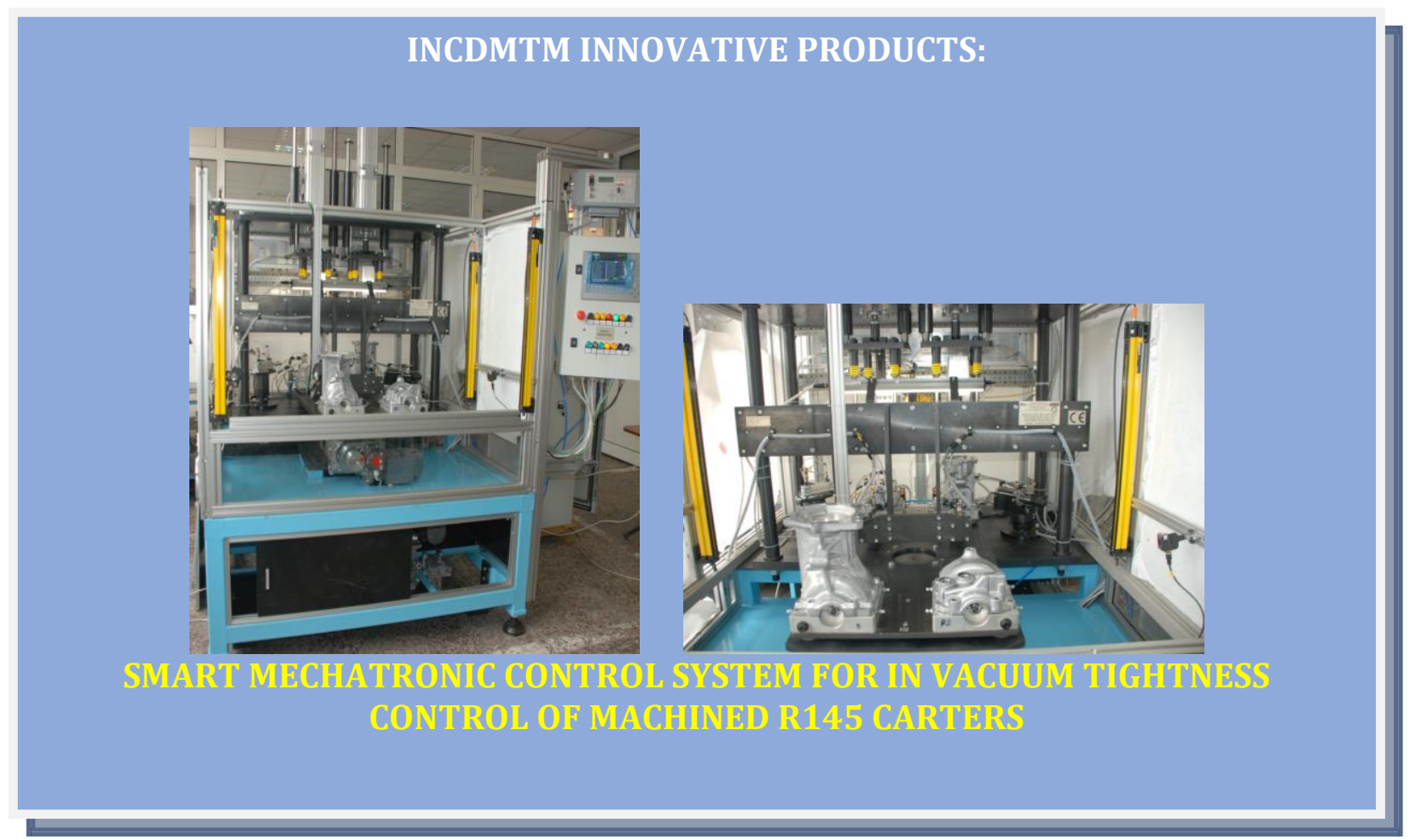

